\title{
First Report of Chlamydia abortus in Farmed Fur Animals
}

\author{
Zhaocai Li (D), $^{1}$ Ping Liu (D), ${ }^{1,2}$ Xiaoan Cao ${ }^{D}$, ${ }^{1}$ Zhongzi Lou, \\ Kinga Zaręba-Marchewka, ${ }^{3}$ Monika Szymańska-Czerwińska, ${ }^{3,4}$ Krzysztof Niemczuk, \\ Bo Hu ${ }^{(D)},{ }^{5}$ Xue Bai $(\mathbb{D})^{5}$ and Jizhang Zhou $\mathbb{D}^{1}$ \\ ${ }^{1}$ State Key Laboratory of Veterinary Etiological Biology, Lanzhou Veterinary Research Institute, \\ Chinese Academy of Agricultural Sciences, Lanzhou, China \\ ${ }^{2}$ Center for Biomedical Research, Northwest Minzu University, Lanzhou, China \\ ${ }^{3}$ Department of Cattle and Sheep Diseases, National Veterinary Research Institute, Pulawy, Poland \\ ${ }^{4}$ Laboratory of Serological Diagnosis, National Veterinary Research Institute, Pulawy, Poland \\ ${ }^{5}$ Institute of Special Animal and Plant Sciences, Chinese Academy of Agricultural Sciences, Changchun, China
}

Correspondence should be addressed to Xue Bai; xuebai_1982@126.com and Jizhang Zhou; zhoujizhang@caas.cn

Received 8 September 2018; Revised 6 November 2018; Accepted 12 November 2018; Published 26 November 2018

Academic Editor: Xiaofeng Fan

Copyright (c) 2018 Zhaocai Li et al. This is an open access article distributed under the Creative Commons Attribution License, which permits unrestricted use, distribution, and reproduction in any medium, provided the original work is properly cited.

Chlamydia (C.) abortus, a globally distributed obligate intracellular bacterium, has attracted increasing interest according to its veterinary importance and zoonotic nature. C. abortus can infect a variety of animals and cause foetal loss in livestock resulting in economic loss. In this study, the samples collected from two farms of foxes $(n=20)$, raccoon dogs $(n=15)$ and minks $(n=20)$, were investigated by Chlamydiaceae- and Chlamydia species-specific real-time PCR. The results showed that all the tested foxes (20/20) and raccoon dogs (15/15) harbored Chlamydia spp., while 5\% of minks (1/20) were positive for Chlamydia spp. C. abortus was identified in all positive samples as the dominant Chlamydia species, with C. pecorum DNA coexistence in some of the rectal samples (7/20) taken from foxes. Phylogenetic analysis based on specific gene fragments of 16S rRNA, IGS-23S rRNA, and ompA revealed that all sequences obtained in this study were assigned to the Chlamydiaceae family with high similarity to C. abortus S26/3 and B577 previously identified in ruminants. This is the first report confirming that farmed foxes, raccoon dogs, and minks carry C. abortus. Further studies are needed to fully elucidate the epidemiology and pathogenicity of this pathogen in farmed fur animals as well as the potential risks to public health.

\section{Introduction}

Chlamydia spp. are obligate intracellular bacteria widely distributed throughout the world and responsible for a variety of diseases in humans and animals. To date, eleven species have been identified along with five newly characterized candidate species in the single genus of the family Chlamydiaceae [1-5]. Chlamydia abortus (C. abortus) has attracted increasing interest due to its veterinary importance and zoonotic potential. C. abortus can infect a variety of mammalian hosts and is associated with abortion cases in ruminants, pigs, horses, rabbits, guinea pigs, and mice [6]. In addition, infection with $C$. abortus in nonmammalian hosts has also been reported recently [7, 8]. Infections of C. abortus are common in ruminants such as sheep, goats, and cattle in many countries around the world, causing tremendous economic loss for the livestock industry [9]. Infected animals may be a source of infection in humans and lead to severe outcomes including respiratory disorder and miscarriage $[10,11]$. The wide distribution, broad-host infection, and zoonotic potential of C. abortus may exhibit an increasing but neglected risk to public health. Therefore, there is an urgent need to investigate the presence of this zoonotic pathogen in animals closely related to humans and evaluate the potential health risk.

Foxes, raccoon dogs, and minks are captive-bred wild animals raised as fur animals with substantial populations in northern Europe, northern America, and China [12]. These animals are suspected to be infected with a wide range of pathogens including several important zoonotic ones, such 
as rabies virus, hepatitis E virus, and influenza virus [1315]. However, there are as yet no reports on the prevalence of chlamydiosis in these animals. Indeed, reproductive failure cases of abortion and stillbirth in foxes, raccoon dogs, and minks were commonly observed in some farms with unknown reasons in Northeast China. Thus, a survey was conducted in China to acquire information concerning farmed fur animal species as possible reservoirs of Chlamydia spp.

\section{Materials and Methods}

Samples from fur animals came from two farms located in the suburbs of Changchun city, Jilin province, China, and sampling was performed in November 2017 in a local slaughterhouse. All samples were collected before the slaughtering of the animals and immediately transported on ice to the laboratory. A formal ethical approval is not required for this kind of study and sampling was performed by authorized veterinarians during routine medical and veterinary activities. A total of 55 sampled animals included 9 male and 11 female foxes, 12 male and 3 female raccoon dogs, and 16 male and 4 female minks (Table 1). In total, rectal swabs $(n=55)$, whole blood $(n=55)$ samples, conjunctive swabs $(n=35)$, and vaginal swabs $(n=18)$ were collected from apparently healthy minks, while the foxes and raccoon dogs came from farm with an abortion history. Samples were stored at $-80^{\circ} \mathrm{C}$ until use. DNA extraction from blood $(200 \mu \mathrm{l})$, vaginal swabs, and conjunctive swabs was performed using the commercial TIANamp Genomic DNA Kit (Tiangen Biotech Co., LTD) following the manufacturer's protocol. For rectal swabs, the QIAamp DNA Stool Mini Kit (Qiagen) was used for DNA extraction. All DNA samples were eluted in $100 \mu \mathrm{l}$ of elution buffer and screened using a Chlamydiaceae-specific real-time PCR targeting the 23S rRNA gene fragment, as previously described by Ehricht et al. [16]. All Chlamydiaceae-positive samples were retested with specific real-time PCR assays to identify Chlamydia species including C. abortus, C. pecorum, C. psittaci, C. felis, and C. suis [17]. An analytical cut-off value of 39.0 was selected corresponding to the defined lower limit of detection of the test. Any Ct value above this defined limit would, thereafter, be considered unreliable. For further quantification DNA concentration from the positive samples, the target fragments for each detecting primer pairs were chemically synthesized and cloned into pMD18T vector (Takara). Quantification of the recombinant plasmids was done on a Nanodrop ND-2000 (Thermo, US), and 10 -fold dilutions $\left(6 \times 10^{7}\right.$ copies to $6 \times 10^{1}$ copy/ $\left.\mu \mathrm{l}\right)$ were used as positive controls to establish a standard curve for quantification. The Chlamydia DNA copies in the samples were tested and calculated according to the formulation obtained from the standard curves. Chlamydiaceae positive DNA samples with a high copies based on real-time PCR were used for DNA sequencing to confirm the identity of Chlamydia spp. Specific fragments of ompA, 16S rRNA, and $16 \mathrm{~S}$ rRNA-23S rRNA intergenic spacer together with 23S rRNA domain I (IGS-23S rRNA) were amplified with previously published primer sets [8]. PCR amplicons were sent to an external company (Shanghai Sangon Biotech, China) for sequencing. The obtained sequences were deposited in the GenBank database with the following accession numbers: MH532474-MH532478 (16S rRNA), MH537631-MH537633 (IGS-23S rRNA), and MH542157-MH542161 (ompA).

The data were analysed using MEGA 5.05 software [18]. Amplicons were subjected to BLAST analysis against the GenBank database (NCBI) to identify related entries and aligned with a panel of Chlamydia reference strains. To assess the phylogenetic relationship between Chlamydia spp. and the tested samples, phylogenetic trees for 16S rRNA (1358bp) and IGS-23S rRNA (990 bp) as well as ompA (940 $\mathrm{bp)}$ were constructed by the neighbor-joining method with 1,000 replicates' bootstrap using the Maximum Composite Likelihood model with MEGA 5.05.

\section{Results and Discussion}

The results of real-time PCR detection are presented in Table 1 and Table S1. Among 55 tested animals, 36 were shedders of Chlamydiaceae giving a prevalence rate of $65.45 \%$. Foxes and raccoon dogs demonstrated the highest level of Chlamydiaceae prevalence at $100 \%$. Positive results for Chlamydiaceae were obtained for all rectal swabs from foxes and raccoon dogs irrespective of gender. DNA of Chlamydiaceae was also present in several blood $(n=10)$ and conjunctive swab $(n=24)$ samples from foxes and raccoon dogs. For the females, vaginal swab samples revealed a high rate of positive results for Chlamydiaceae in foxes (9/11) and raccoon dogs (1/3). Further testing with species-specific real-time PCR identified the detected agent in all Chlamydiaceae positive samples as C. abortus. Moreover in 7 rectal swabs from foxes the coexistence of C. pecorum was confirmed. No other Chlamydia species was identified. Only one rectal swab from a male mink was tested positive for Chlamydiaceae and determined as $C$. abortus with a relatively low level of DNA copies.

To further confirm the presence of Chlamydia spp., amplification and sequencing of $16 \mathrm{~S}$ rRNA, IGS-23S rRNA, and $\operatorname{ompA}$ gene fragments were carried out on high positive samples. The $16 \mathrm{~S}$ rRNA gene amplicons were successfully obtained from 2 samples originating from foxes (MH532477, MH532478) and 3 samples originating from raccoon dogs (MH532474-MH532476), IGS-23S rRNA from 1 fox (MH537633) and 2 samples from raccoon dogs (MH537631, MH537632), and ompA from 1 fox (MH542161) and 4 samples from raccoon dogs (MH542157-MH542160), while the process of obtaining amplicons from mink samples failed. Dendrograms were constructed on the basis of ompA, $16 \mathrm{~S}$ rRNA, and IGS-23S rRNA gene fragments aligning with Chlamydia species reference sequences available in GenBank and altogether showed a similar topology. All sequences obtained in this study were assigned to C. abortus within the Chlamydiaceae family (Figure 1). In addition, phylogenetic analysis showed ompA sequences were clustered with the strains C. abortus $\mathrm{S} 26 / 3$ and B577 hosted by ruminants as the closest relative.

Chlamydiosis is common in livestock, poultry, companion, and wild animals $[19,20]$. In the available literature, there is a lack of reports about chlamydiosis cases in fur animals. In this study, evidence for the existence of C. abortus in farmed 
BioMed Research International

3

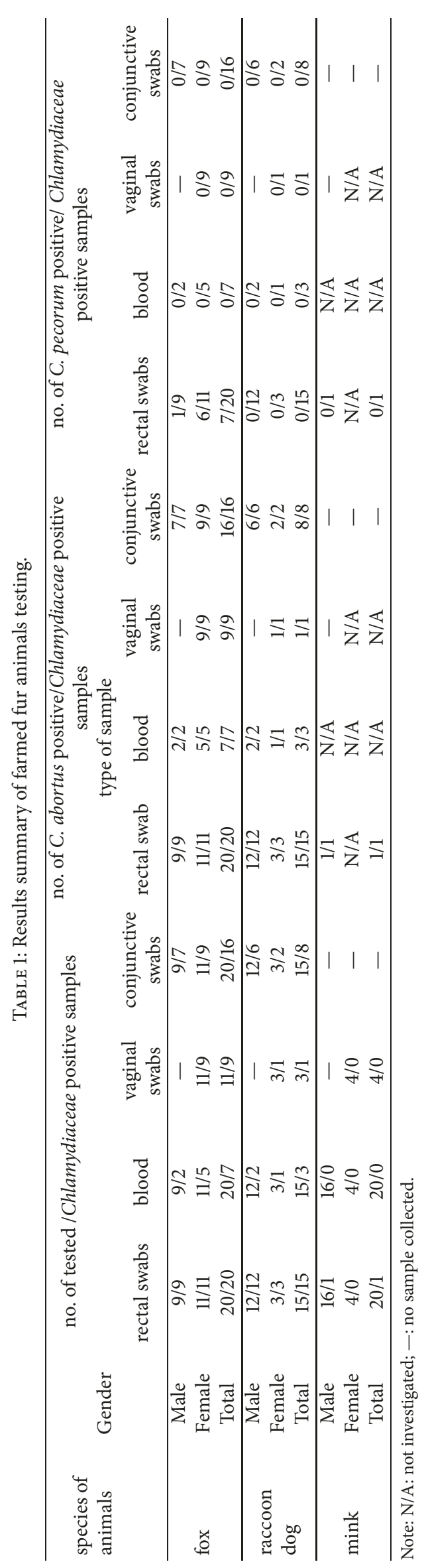




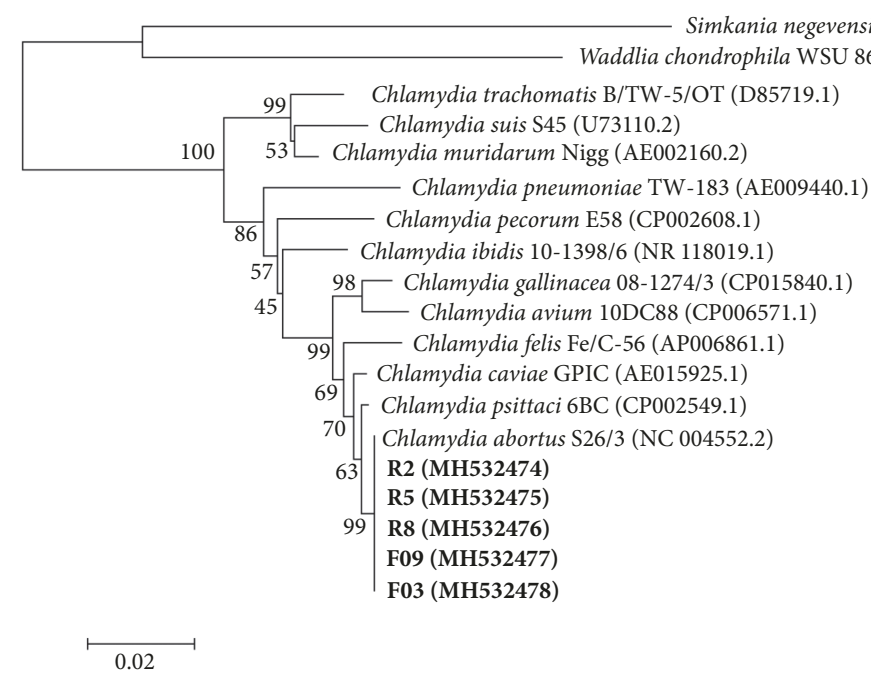

(a)

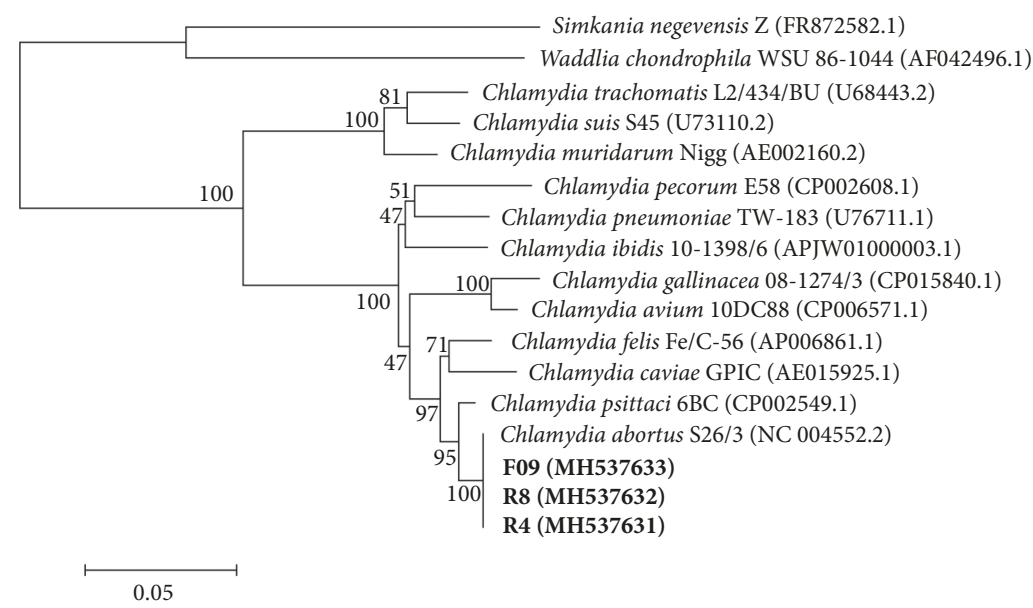

(b)

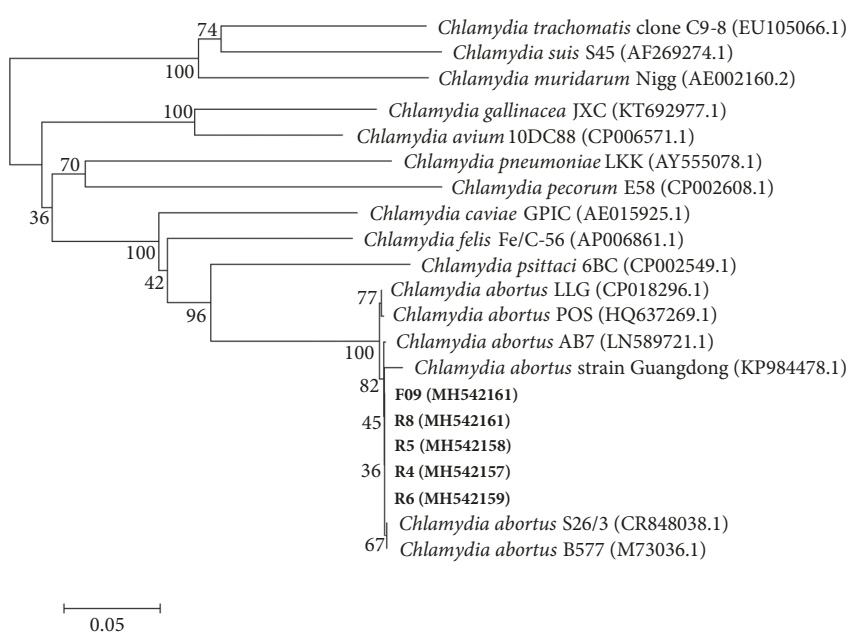

(c)

FIGURE 1: Phylogenetic tree based on 16S rRNA gene fragment (1358 bp) (a), 16S-23S intergenic spacer and full length of 23S rRNA domain I fragment (990 bp) (b), and ompA gene fragment (940 bp) (c). Representative sequences of established Chlamydiaceae species as well as the sequences obtained from this study (Genbank accession number was shown in bold) were included. S. negevensis strain $\mathrm{Z}$ was used as an outgroup. Based on these alignments, phylogenetic trees were constructed by the neighbor-joining method using the Maximum Composite Likelihood model with MEGA 5.05. 
fur animals was shown for the first time. It seemed that the gastrointestinal tract provided a major endemic habitat for C. abortus in farmed foxes and raccoon dogs, although the genital tract was generally considered as the colonized site for this pathogen in female animals. Another Chlamydia species found in this study as a coexisting pathogen in a few rectal swabs in foxes was C. pecorum, pathogen of the koala, and commonly found in the gastrointestinal tract of cattle [21, 22]. These results suggested that $C$. abortus was the dominant Chlamydia species carried by tested animals. However, due to the limited number of samples investigated in this study, more evidence is required to reveal the relationship between C. abortus infection and reproductive disorder in foxes and raccoon dogs. In addition, it is not clear whether fur animals are the natural hosts of Chlamydia spp. such as C. abortus or if infection occurs via other vectors. It is worth mentioning that the feed for fur animals in China always contains fish, chicken, and occasionally pig products. As has been reported, the prevalence of Chlamydia spp. in chickens and pigs is quite common in China [23, 24]. Therefore, feeding should be considered as a possible way for farmed fur animals to become infected with Chlamydia spp.

C. abortus is well known for its zoonotic nature. Frequent human contact with domestic livestock at work increases the risk of infection. According to data published in 2002, the average number of notified cases of human chlamydiosis originating from $C$. abortus infected animals was approximately 100 per year in Germany [25]. Although the possible transmission of $C$. abortus from farmed fur animals to humans has not been investigated yet, environmental contamination by the faecal shedding of this zoonotic agent may become a potential source of infection for workers exposed to infected animals during their daily activities.

\section{Conclusions}

This is the first report confirming that farmed fur animals, foxes, raccoon dogs, and minks, mainly harbored C. abortus, while C. pecorum occasionally coexists in foxes. Further studies are needed to fully elucidate the epidemiology and pathogenicity of C. abortus in farmed fur animals. Moreover, an evaluation of the potential health risk to fur farm personnel and the creation of a strategy to preserve human safety are required.

\section{Data Availability}

The data used to support the findings of this study are available from the corresponding author upon request.

\section{Conflicts of Interest}

The authors declare that no conflicts of interest exist.

\section{Acknowledgments}

This work was supported by NSFC grant (31502081), the Central Public-interest Scientific Institution Basal Research Fund (1610312016024), National Key R\&D program of China
(2017YFD0500905), and basic research expenses covered by the Chinese Academy of Agricultural Sciences (Y2017CG34) and also funded by KNOW (Leading National Research Centre) Scientific Consortium "Healthy Animal - Safe Food," decision of Ministry of Science and Higher Education (051/KNOW2/2015).

\section{Supplementary Materials}

Table S1: summary of real-time PCR results. (Supplementary Materials)

\section{References}

[1] K. Sachse, P. M. Bavoil, B. Kaltenboeck et al., "Emendation of the family Chlamydiaceae: Proposal of a single genus, Chlamydia, to include all currently recognized species," Systematic and Applied Microbiology, vol. 38, no. 2, pp. 99-103, 2015.

[2] F. Vorimore, R.-C. Hsia, H. Huot-Creasy et al., "Isolation of a New Chlamydia species from the Feral Sacred Ibis (Threskiornis aethiopicus): Chlamydia ibidis," PLoS ONE, vol. 8, no. 9, 2013.

[3] A. Taylor-Brown, N. L. Bachmann, N. Borel, and A. Polkinghorne, "Culture-independent genomic characterisation of Candidatus Chlamydia sanzinia, a novel uncultivated bacterium infecting snakes," BMC Genomics, vol. 17, no. 1, 2016.

[4] A. Taylor-Brown, L. Spang, N. Borel, and A. Polkinghorne, "Culture-independent metagenomics supports discovery of uncultivable bacteria within the genus Chlamydia," Scientific Reports, vol. 7, no. 1, 2017.

[5] E. Staub, H. Marti, R. Biondi et al., "Novel Chlamydia species isolated from snakes are temperature-sensitive and exhibit decreased susceptibility to azithromycin," Scientific Reports, vol. 8, no. 1, 2018.

[6] K. D. E. Everett, R. M. Bush, and A. A. Andersen, "Emended description of the order Chlamydiales, proposal of Parachlamydiaceae fam. nov. and Simkaniaceae fam. nov., each containing one monotypic genus, revised taxonomy of the family Chlamydiaceae, including a new genus and five new species, and standards for the identification of organisms," International Journal of Systematic Bacteriology, vol. 49, no. 2, pp. 415-440, 1999.

[7] M. Szymańska-Czerwińska, A. Mitura, K. Zaręba, C. Schnee, A. Koncicki, and K. Niemczuk, "Poultry in Poland as Chlamydiaceae carrier," Journal of Veterinary Research (Poland), vol. 61, no. 4, pp. 411-419, 2017.

[8] M. Szymańska-Czerwińska, A. Mitura, K. Niemczuk et al., "Dissemination and genetic diversity of chlamydial agents in Polish wildfowl: Isolation and molecular characterisation of avian Chlamydia abortus strains," PLoS ONE, vol. 12, no. 3, 2017.

[9] D. Longbottom and L. J. Coulter, "Animal chlamydioses and zoonotic implications," Journal of Comparative Pathology, vol. 128, no. 4, pp. 217-244, 2003.

[10] G. Walder, H. Hotzel, C. Brezinka et al., "An unusual cause of sepsis during pregnancy: recognizing infection with Chlamydophila abortus," Obstetrics \& Gynecology, vol. 106, no. 5, pp. 1215-1217, 2005.

[11] N. Ortega, M. R. Caro, M. C. Gallego et al., "Isolation of Chlamydia abortus from a laboratory worker diagnosed with atypical pneumonia," Irish Veterinary Journal, vol. 69, no. 1, 2016.

[12] The welfare of animals kept for fur production. Report on the Scientific Committee on Animal Health and Animal Welfare 
Adopted on 12-13 December 2001. https://ec.europa.eu/food/ sites/food/files/safety/docs/sci-com_scah_out67_en.pdf.

[13] Y. Liu, S. Zhang, J. Zhao et al., "Fox- and raccoon-dogassociated rabies outbreaks in northern China," Virologica Sinica, vol. 29, no. 5, pp. 308-310, 2014.

[14] J. S. Krog, S. Ø. Breum, T. H. Jensen, and L. E. Larsen, "Hepatitis E virus variant in farmed mink, Denmark," Emerging Infectious Diseases, vol. 19, no. 12, pp. 2028-2030, 2013.

[15] W. Jiang, S. Wang, C. Zhang et al., "Characterization of H5N1 highly pathogenic mink influenza viruses in eastern China," Veterinary Microbiology, vol. 201, pp. 225-230, 2017.

[16] R. Ehricht, P. Slickers, S. Goellner, H. Hotzel, and K. Sachse, "Optimized DNA microarray assay allows detection and genotyping of single PCR-amplifiable target copies," Molecular and Cellular Probes, vol. 20, no. 1, pp. 60-63, 2006.

[17] A. Pantchev, R. Sting, R. Bauerfeind, J. Tyczka, and K. Sachse, "Detection of all Chlamydophila and Chlamydia spp. of veterinary interest using species-specific real-time PCR assays," Comparative Immunology, Microbiology \& Infectious Diseases, vol. 33, no. 6, pp. 473-484, 2010.

[18] K. Tamura, D. Peterson, N. Peterson, G. Stecher, M. Nei, and S. Kumar, "MEGA5: molecular evolutionary genetics analysis using maximum likelihood, evolutionary distance, and maximum parsimony methods," Molecular Biology and Evolution, vol. 28, no. 10, pp. 2731-2739, 2011.

[19] A. Pospischil, "From diseaseto etiology: Historical aspects of Chlamydia-related diseases in animals and humans," Drugs of Today, vol. 45, pp. 141-146, 2009.

[20] N. Borel, A. Polkinghorne, and A. Pospischil, "A Review on Chlamydial Diseases in Animals: Still a Challenge for Pathologists?” Veterinary Pathology, vol. 55, no. 3, pp. 374-390, 2018.

[21] A. R. Legione, J. Amery-Gale, M. Lynch et al., "Chlamydia pecorum infection in free-ranging koalas (Phascolarctos cinereus) on French Island, Victoria, Australia," Journal of Wildlife Diseases, vol. 52, no. 2, pp. 426-429, 2016.

[22] J. Li, W. Guo, B. Kaltenboeck et al., "Chlamydia pecorum is the endemic intestinal species in cattle while C. gallinacea, $C$. psittaci and C. pneumoniae associate with sporadic systemic infection," Veterinary Microbiology, vol. 193, pp. 93-99, 2016.

[23] W. Guo, J. Li, B. Kaltenboeck, J. Gong, W. Fan, and C. Wang, "Chlamydia gallinacea, not C. psittaci, is the endemic chlamydial species in chicken (Gallus gallus)," Scientific Reports, vol. 6, no. 1, 2016.

[24] M. Li, M. Jelocnik, F. Yang et al., "Asymptomatic infections with highly polymorphic Chlamydia suis are ubiquitous in pigs," BMC Veterinary Research, vol. 13, no. 1, 2017.

[25] K. Sachse and E. Grossmann, "Chlamydial diseases of domestic animals-zoonotic potential of the agents and diagnostic issues," Dtsch Tierarztl Wochenschr, vol. 109, no. 4, pp. 142-148, 2002. 


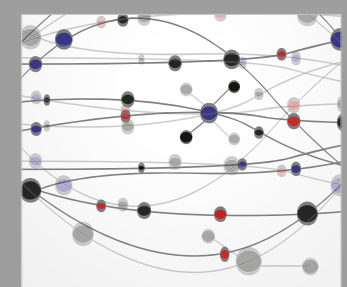

The Scientific World Journal
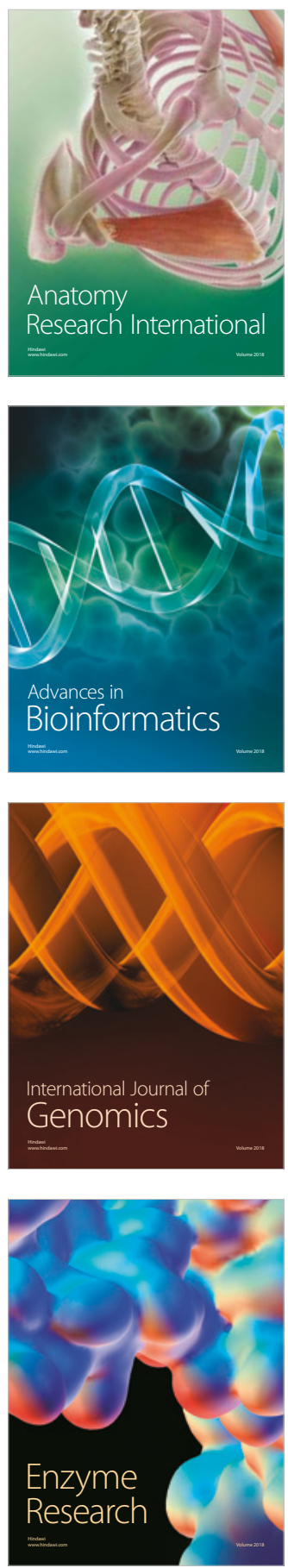
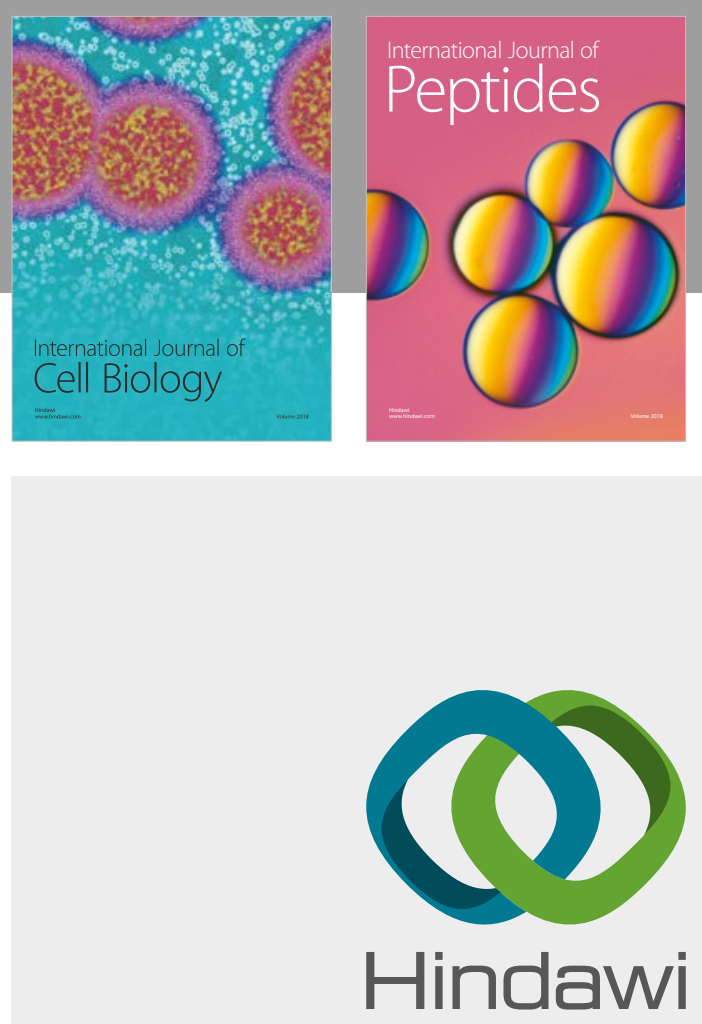

Submit your manuscripts at

www.hindawi.com
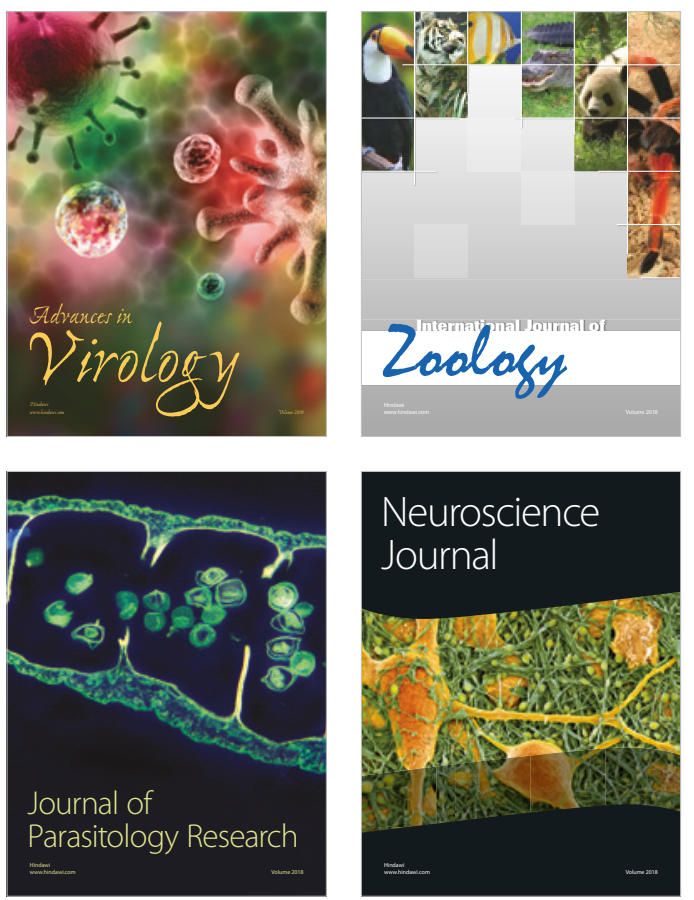
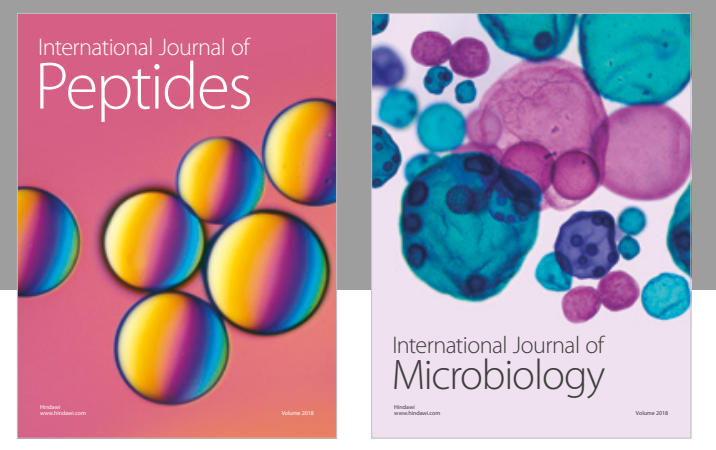

nternational Journal of Microbiology
Journal of
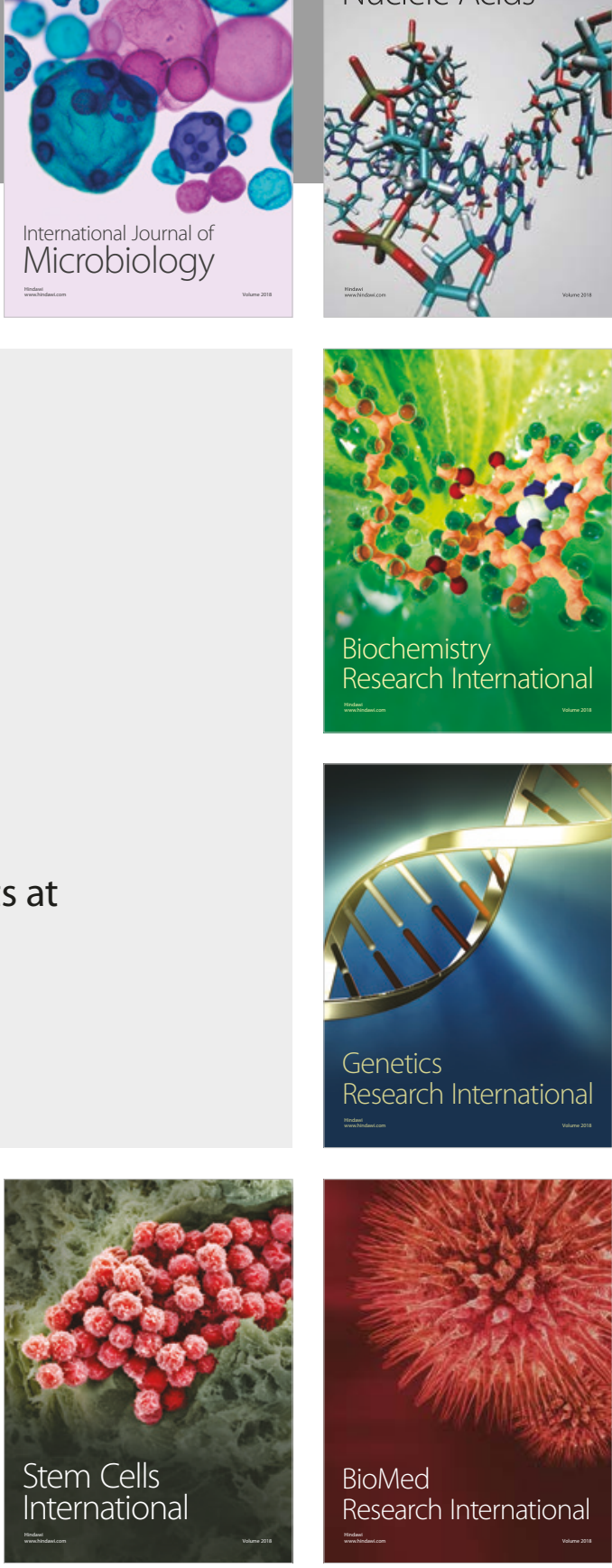
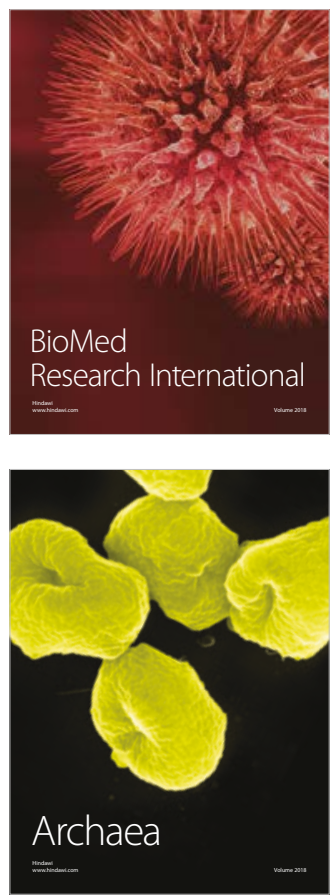